\title{
Role of endoscopic ultrasonography for differential diagnosis of upper gastrointestinal submucosal lesions
}

\author{
Qian Su' ${ }^{1}$ Jin Peng ${ }^{2}$, Xiong Chen ${ }^{1}$, Zhiming Xiao' ${ }^{1}$, Rui Liu ${ }^{1}$ and Fen Wang ${ }^{1 *}$
}

\begin{abstract}
Objective: To determine the accuracy of endoscopic ultrasonography (EUS) in the diagnosis of upper gastrointestinal submucosal lesions (SMLs).

Methods: This was a retrospective study involving patients diagnosed with SMLs using EUS and confirmed by histopathology from November 2014 to December 2020 at The Third Xiangya Hospital of Central South University.

Results: A total of 231 patients with SMLs were examined by EUS. Histologically, 107 lesions were stromal tumors, and 75 lesions were leiomyomas. Stromal tumors were mainly located in the stomach (89.7\%), and leiomyomas were predominantly seen in the esophagus (69.3\%). The diagnostic accuracy of EUS for stromal tumors and leiomyomas was $80.4 \%$ and $68.0 \%$, respectively. The diagnostic accuracy was highest for lesions located in the muscularis mucosa. The mean diameter of stromal tumors measured using EUS was significantly larger than that of leiomyomas ( $21.89 \mathrm{~mm}$ vs. $12.35 \mathrm{~mm}, p<0.001)$. Stromal tumors and leiomyomas originated mainly from the muscularis propria (94.4\%) and the muscularis mucosa (56.0\%), respectively. Compared with the very low-risk and low-risk groups of stromal tumors according to the National Institute of Health guidelines, the intermediate-risk and high-risk groups were more likely to have a lesion $>3 \mathrm{~cm}(p<0.001)$ and a surface ulcer $(p<0.01)$ identified by EUS.

Conclusions: EUS has good diagnostic value for the diagnosis of upper gastrointestinal SMLs based on the lesion size and the muscle layer of origin. The diagnostic accuracy of EUS lesions is related to the origin, and the diagnostic accuracy is greatest in the mucosal muscularis layer. Stromal tumors $>3 \mathrm{~cm}$ and a surface ulcer on EUS are likely to be intermediate or high risk for invasion.
\end{abstract}

Keywords: Endoscopic ultrasonography, Upper gastrointestinal submucosal lesion, Stromal tumor, Leiomyoma, Invasive risk

\section{Introduction}

Submucosal lesions (SMLs) in the digestive tract refer to abnormal lesions originating from the various layers under the mucosa, and upper gastrointestinal SMLs

\footnotetext{
*Correspondence: wfen-judy@csu.edu.cn

${ }^{1}$ Department of Gastroenterology, Hunan Key Laboratory

of Non-Resolving Inflammation and Cancer, The Third Xiangya Hospital of Central South University, 138 Tongzi Road, Changsha 410013, Hunan, People's Republic of China

Full list of author information is available at the end of the article
}

are the most common. Differential diagnosis of the various types of SMLs during routine endoscopy is difficult [1]. Different pathological types of SMLs have various biological behaviors. Pathologically, they can be divided into benign, malignant, and potentially malignant categories. Therefore, it is important to distinguish the different pathological types of upper gastrointestinal SMLs. Endoscopic ultrasonography (EUS) is a very useful tool for the evaluation of these upper gastrointestinal SMLs. Not only can it be used to observe the morphological structure of 
the lesions in the cavity, but EUS can also perform realtime scanning to obtain information regarding the wall layer structure of the digestive tract, the originating layer of the lesions, and the relationship between the extent of lesion infiltration and the surrounding tissues, peripheral lymph nodes and adjacent organs. EUS-guided fineneedle aspiration/biopsy (EUS-FNA/FNB) can further help to obtain cells or tissues for pathological confirmation $[2,3,7]$. In this study, we reviewed and compared the distribution, size, endoscopic features, and pathological results of SMLs to determine the diagnostic value of EUS for SMLs. This was performed by analyzing the diagnostic consistency rate, the relationship between endoscopic features and invasion risk, and the relationships among the distribution, diameter, origin and pathological properties of the lesions.

\section{Materials and methods \\ Patient selection}

In this retrospective study, adult patients with SMLs diagnosed and treated at The Third Xiangya Hospital of Central South University from November 2014 to December 2020 were included and their clinical data were retrieved. The study was approved by the Ethics Committee of The Third Xiangya Hospital of Central South University. All patients gave written informed consent for the endoscopic and surgical procedures. Patients were excluded if they were under 18 years of age, had incomplete endoscopic or pathological data, or were pregnant.

All patients underwent a routine endoscopy and EUS to confirm the location of the SML in the upper gastrointestinal tract. Six patients underwent EUS-FNA/FNB prior to surgery, 14 patients underwent EUS elastography, and one patient underwent contrast-enhanced EUS. Meanwhile, all patients underwent an endoscopic or surgical resection. Postoperative specimens were collected for pathological and immunohistochemical examination.

\section{Instruments and procedures}

After eight hours of fasting before the procedure and obtaining written informed consent, oral gel was applied for local anesthesia and intravenous propofol was administered for sedation. A mini-probe $(20 \mathrm{MHz}$; SP-701, Fujiron, Tokyo, Japan) or linear array ultrasound probe (6.0-7.5 MHz; EU-ME2, Olympus, Tokyo, Japan) was selectively used according to the findings of the routine endoscopy. The lumen was filled with water for scanning. Once the position of the lesion was identified, the size was measured, and the origin and echo characteristics of the lesion were determined (heterogeneity, whether the boundary was clear, etc.). The ultrasound device was then used in Doppler mode to detect blood flow, velocity, and direction.

\section{Endoscopic ultrasound stratification of the normal digestive tract wall}

According to a study by Kuroki et al. [4], the digestive tract wall can be divided into five layers by EUS. By comparing the results with histological findings, the first hyperechoic layer was identified as the mucosal reflex interface, the second hypoechoic layer was the muscularis mucosa, the third hyperechoic layer was the submucosal layer, the fourth hypoechoic layer was the muscularis propria, and the fifth hyperechoic layer was the serosal layer. The upper and middle third esophageal wall did not have a serosal layer, and the fifth layer was the surrounding connective tissue.

\section{Determination of invasive risk by stromal tumors}

According to the revised recommendations of the National Institute of Health (NIH) standard titled "Consensus for the Diagnosis and Treatment of Gastrointestinal Stromal Tumors in China (2017 Edition)" [5], patients with stromal tumors were divided into one of four groups based on the risk of tumor invasion, including very low-risk (VLR), low-risk (LR), intermediate-risk (IR) and high-risk (HR).

\section{Statistical analysis}

Statistical analyses were performed with SPSS version 25.0 (IBM). Quantitative data were shown as mean \pm standard deviation (SD) and were compared using the $t$-test or Mann-Whitney $U$ test. The chisquare test or Fisher's exact test were used to compare categorical data. A $p$ value less than 0.05 was considered statistically significant.

\section{Results \\ Clinical data}

A total of 231 patients with SMLs diagnosed during the study period were included for analysis. There were 122 males and 109 females with a mean age of $51.89 \pm 11.91$ years (range: $22-79$ years). The most common site of esophageal lesions was the upper and middle third of the esophagus while that of the stomach was the gastric fundus (Table 1). The most common histological types of SMLs in the esophagus and stomach were leiomyomas and stromal tumors, respectively (Table 2; Figs. 1, 2). The other SMLs observed in the study patients were schwannoma (Fig. 3), heterotopic pancreas (Fig. 4), benign cyst (Fig. 5), glomus tumor (Fig. 6), hamartoma (Fig. 7), solitary fibroma (Fig. 8), haemo-lymphangioma (Fig. 9) and angiogenic tumor (Fig. 10). The most common sites of origin of stromal tumors, leiomyomas and ectopic pancreas were the 
Table 1 Distribution of lesions

\begin{tabular}{lll}
\hline Origin & $\begin{array}{l}\text { Number of } \\
\text { cases }\end{array}$ & Percentage \\
\hline Esophagus & 63 & 27.3 \\
Upper esophagus & 23 & 10.0 \\
Mid-esophagus & 22 & 9.5 \\
Lower esophagus & 15 & 6.5 \\
Multiple locations & 3 & 1.3 \\
Stomach & 159 & 68.8 \\
Gastric angle & 3 & 1.3 \\
Junction between antrum and body & 4 & 1.7 \\
Cardia & 5 & 2.2 \\
Junction between fundus and body & 6 & 2.6 \\
Gastric antrum & 14 & 6.1 \\
Gastric body & 45 & 19.5 \\
Gastric fundus & 82 & 35.5 \\
Duodenum & 9 & 3.9 \\
Descending duodenum & 5 & 2.2 \\
Duodenal bulb & 4 & 1.7 \\
Total & 231 & 100 \\
\hline
\end{tabular}

muscularis propria, muscularis mucosa, and submucosa, respectively (Table 3 ).

\section{Diagnostic accuracy of EUS}

The diagnostic accuracy of EUS for stromal tumors was $80.4 \%$ and for leiomyomas was $68.0 \%$ (Table 4 ). The data were divided into two groups: (A) the EUS diagnosis and pathological diagnosis were the same, and (B) the diagnoses differed. Differences regarding patient age, gender, lesion diameter, location, and origin were compared between the two groups. The results showed that the difference in lesion origin was statistically significant $(p<0.05)$. Furthermore, the diagnostic accuracy of EUS was highest for the lesions located in the muscularis mucosa (muscularis mucosa vs. muscularis propria $p<0.001$; muscularis mucosa vs. submucosa $p<0.001$; muscularis propria vs. submucosa $p=0.001$; Table 5).

\section{Comparison of the maximum diameters of stromal tumors and leiomyomas}

The mean diameter of the stromal tumors as measured by EUS was $21.89 \pm 14.81 \mathrm{~mm}$ (range: $3.80-76.10 \mathrm{~mm}$ ), while that of the leiomyomas was $12.35 \pm 11.18 \mathrm{~mm}$ (range: $2.30-62.00 \mathrm{~mm}$ ) and the difference was statistically significant $(p<0.001)$. The mean diameter of stromal tumors based on the measurement of the surgical specimens was $25.49 \pm 19.49 \mathrm{~mm}$ (range: $5.00-135.00 \mathrm{~mm}$ ), while that of the leiomyomas was $16.65 \pm 13.48 \mathrm{~mm}$ (range: $2.00-60.00 \mathrm{~mm} ; p<0.01$ ).

\section{Comparison of the originating layers of stromal tumors and leiomyomas on EUS}

Most of the stromal tumors originated from the muscularis propria (101/107, 94.4\%), while the leiomyomas mainly originated from the muscularis mucosa $(42 / 75$, $56.0 \%)$. This difference was statistically significant $\left(\chi^{2}=57.66, p<0.001\right)$.

\section{Sensitivity, specificity and predictive value of the originating layer on EUS to determine the pathological nature of the submucosal lesions}

When the originating layer (muscularis propria) of the lesions was used to make a stromal tumor diagnosis, the sensitivity was $94.4 \%$, specificity was $56.0 \%$, positive predictive value was $75.4 \%$ and negative predictive value was

Table 2 Distributions of submucosal lesions (SMLs) in the upper digestive tract based on histopathology

\begin{tabular}{|c|c|c|c|c|c|c|}
\hline Pathological result & Esophagus & Stomach & Duodenum & Total & Percentage & $p$ \\
\hline Stromal tumor & 6 & 96 & 5 & 107 & 46.3 & $0.001^{* *}$ \\
\hline Leiomyoma & 52 & 22 & 1 & 75 & 32.5 & $0.001^{* *}$ \\
\hline Heterotopic pancreas & - & 11 & 2 & 13 & 5.6 & $0.011^{*}$ \\
\hline Schwannoma & - & 11 & - & 11 & 4.8 & 0.080 \\
\hline Inflammation & 1 & 8 & 1 & 10 & 4.3 & 0.222 \\
\hline Benign cyst & 2 & 2 & - & 4 & 1.7 & 0.416 \\
\hline Glomus tumor & - & 4 & - & 4 & 1.7 & 0.640 \\
\hline Angiolipoma & - & 3 & - & 3 & 1.3 & 0.606 \\
\hline Hamartoma & - & 1 & - & 1 & 0.4 & 1.000 \\
\hline Solitary fibroma & 1 & - & - & 1 & 0.4 & 0.311 \\
\hline Haemo-lymphangioma & 1 & - & - & 1 & 0.4 & 0.311 \\
\hline Angiogenic tumor & - & 1 & - & 1 & 0.4 & 1.000 \\
\hline Total & 63 & 159 & 9 & 231 & 100 & \\
\hline
\end{tabular}

***Esophagus, the stomach, and the duodenum have significant differences at the levels of $p<0.05, p<0.01$, respectively 


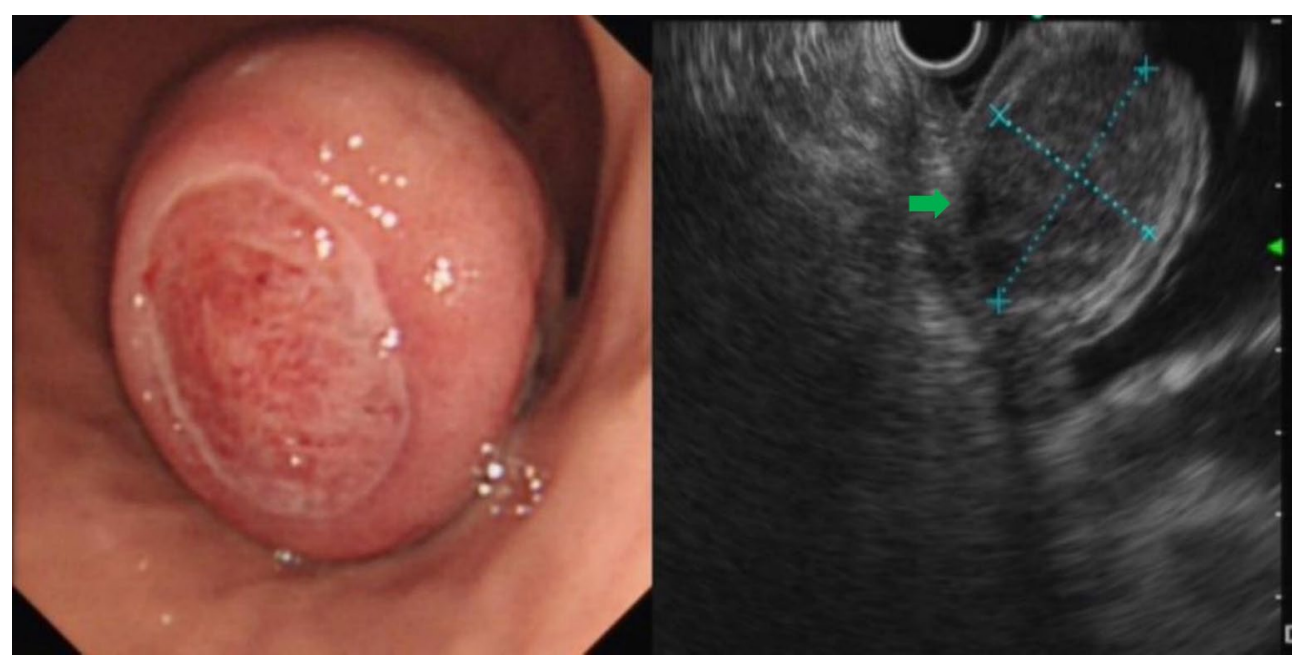

Fig. 1 Stromal tumor of gastric body (green arrow)

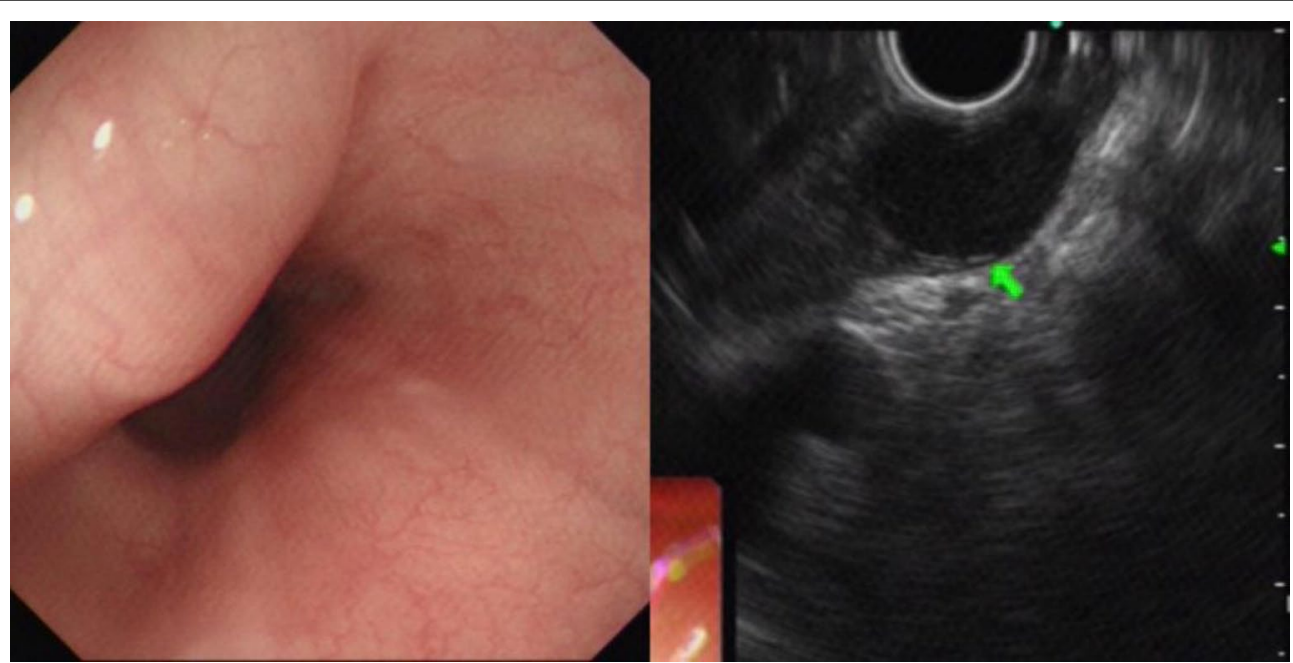

Fig. 2 Leiomyoma of esophagus (green arrow)

87.5\%. Similarly, the sensitivity, specificity, positive predictive value and negative predictive value of the originating layer (muscularis mucosa) of the lesions to make the diagnosis of leiomyoma was $56.0 \%, 94.4 \%, 87.5 \%$ and $75.4 \%$, respectively.

\section{Comparison of the EUS characteristics of stromal tumors with different invasive risks}

One-hundred-and-seven patients with stromal tumors were categorized into either the VLR group (62 patients), LR group (34 patients), IR group (10 patients) or HR group (one patient) according to the revised NIH standards [5]. To compare characteristics, the patients were further grouped into two groups: the VLR + LR group or the IR+HR group. Compared to the VLR+LR group, the IR + HR group was more likely to have a lesion diameter $>3 \mathrm{~cm}(p<0.001)$ as well as a surface ulcer $(p<0.01)$ identified by EUS (Table 6).

\section{Discussion}

SMLs have similar appearances on routine endoscopy irrespective of their histology. Moreover, endoscopic biopsy of SMLs is difficult since a routine endoscopy can only visualize and obtain the normal mucosa on the surface of the lesions. Other imaging methods, such as ultrasound, barium meals, and CT, have low sensitivity and 


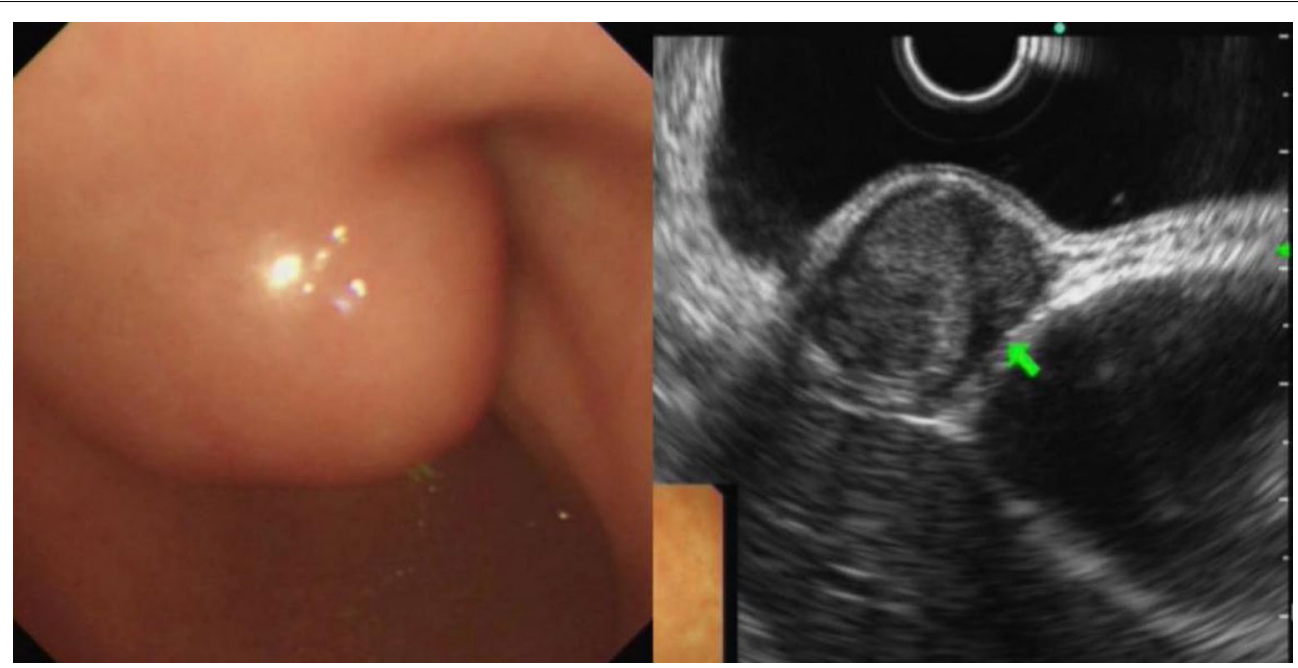

Fig. 3 Schwannoma of gastric body (green arrow)

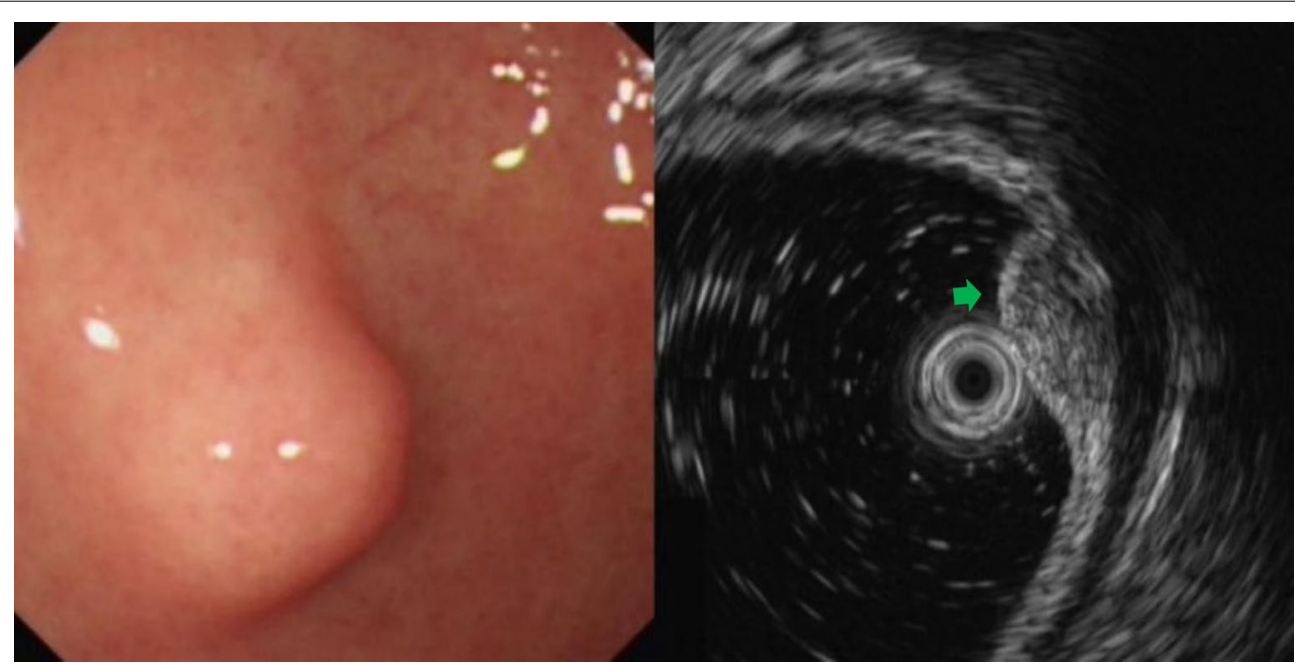

Fig. 4 Heterotopic pancreas of gastric antrum (green arrow)

specificity for the diagnosis of such lesions [6]. EUS can visualize SMLs of the upper digestive tract and provide information regarding the layered structure of the digestive tract wall, the originating layer of the lesions, and the relationship between the lesion and the surrounding tissues, the peripheral lymph nodes and adjacent organs. Moreover, EUS-FNA/FNB can further help to obtain cells or tissues for pathology $[2,3,7]$. Therefore, EUS has become the most effective method for the diagnosis of SML.

In this study, SMLs were found in the stomach (159 patients), esophagus (63 patients), and duodenum (9 patients). The final pathology suggested that stromal tumors and leiomyomas were the most common types of SMLs, consistent with the report by Dias et al. [3]. Stromal tumors were more common in the stomach, while leiomyomas were more common in the esophagus, which is also consistent with previous reports [8].

The postoperative pathological diagnosis was considered the gold standard in this study and was used for comparison with the EUS findings. We found that EUS had good diagnostic value for SMLs. The diagnostic consistency rate of EUS was $80.4 \%$ for stromal tumors and $68.0 \%$ for leiomyomas, similar to previous studies [9]. The rare tumors that were observed, such as glomus tumors and angiolipomas, lacked characteristic features on EUS 


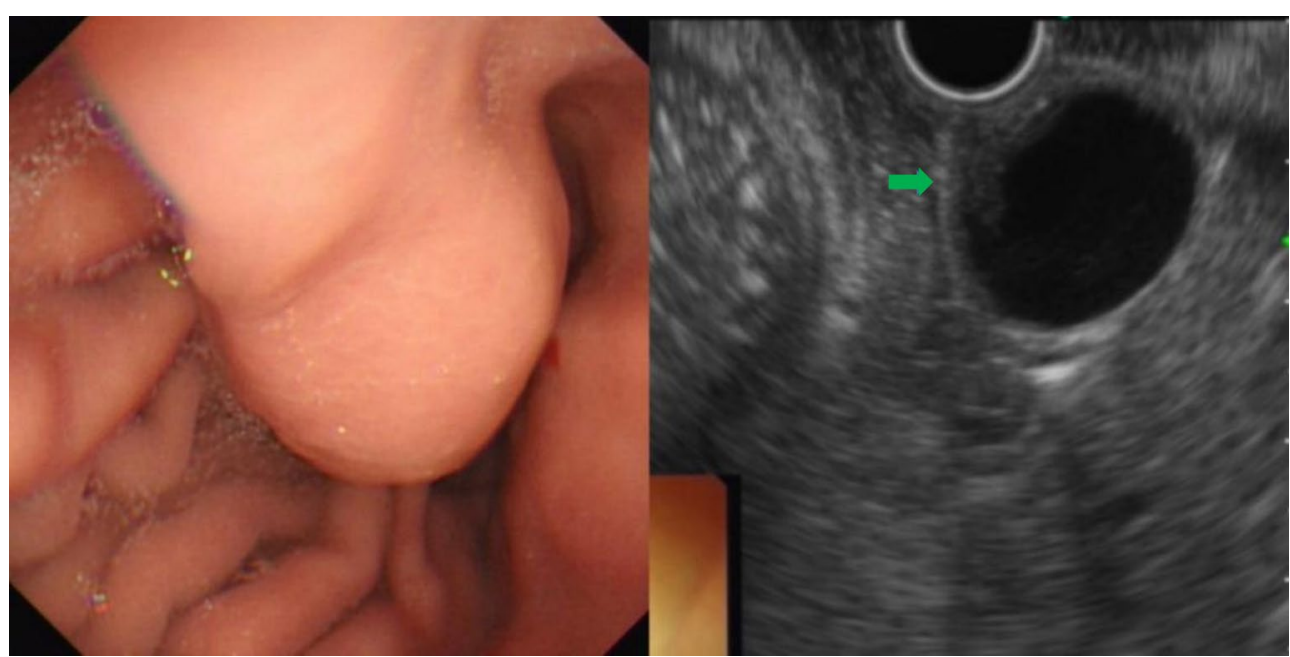

Fig. 5 Benign cyst of gastric body (green arrow)

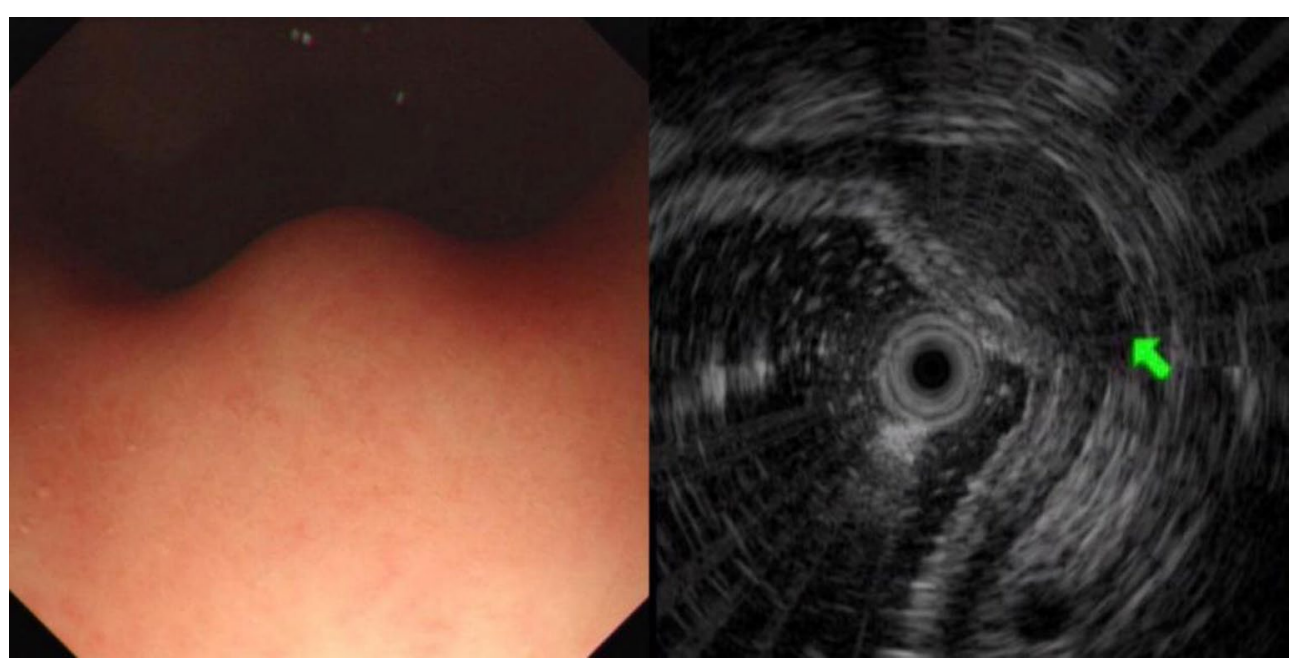

Fig. 6 Glomus tumor of gastric antrum (green arrow)

and appeared similar to common SMLs, such as stromal tumors and leiomyomas, and may be easily misdiagnosed on EUS. Moreover, the accuracy of EUS diagnosis is closely related to the operator's technological and clinical experience, available EUS equipment and the degree of patient cooperation [10]. Therefore, the diagnosis of such diseases needs to be combined with the patient's medical history and other relevant tests, especially pathological examinations. The diagnostic accuracy of lesion type was related to the level of origin, and the diagnostic accuracy of lesions originating from the muscularis mucosa was the greatest, which was consistent with the report by Schulz et al. [11]. This may be due to the many types of submucosal lesions derived from the submucosa and muscularis propria, such as schwannomas, lipomas, ectopic pancreas, and other relatively rare lesions, resulting in insufficient diagnosis experience. Therefore, EUS is reliable for the diagnosis of lesions originating from the muscularis mucosa. For lesions originating from the submucosa or muscularis propria, other auxiliary examinations should be considered or pathology should be obtained for a histological diagnosis.

Additionally, the maximum diameters of the lesions were compared and the results suggested that the mean maximum diameter of stromal tumors was significantly greater than that of leiomyomas. This may provide a 


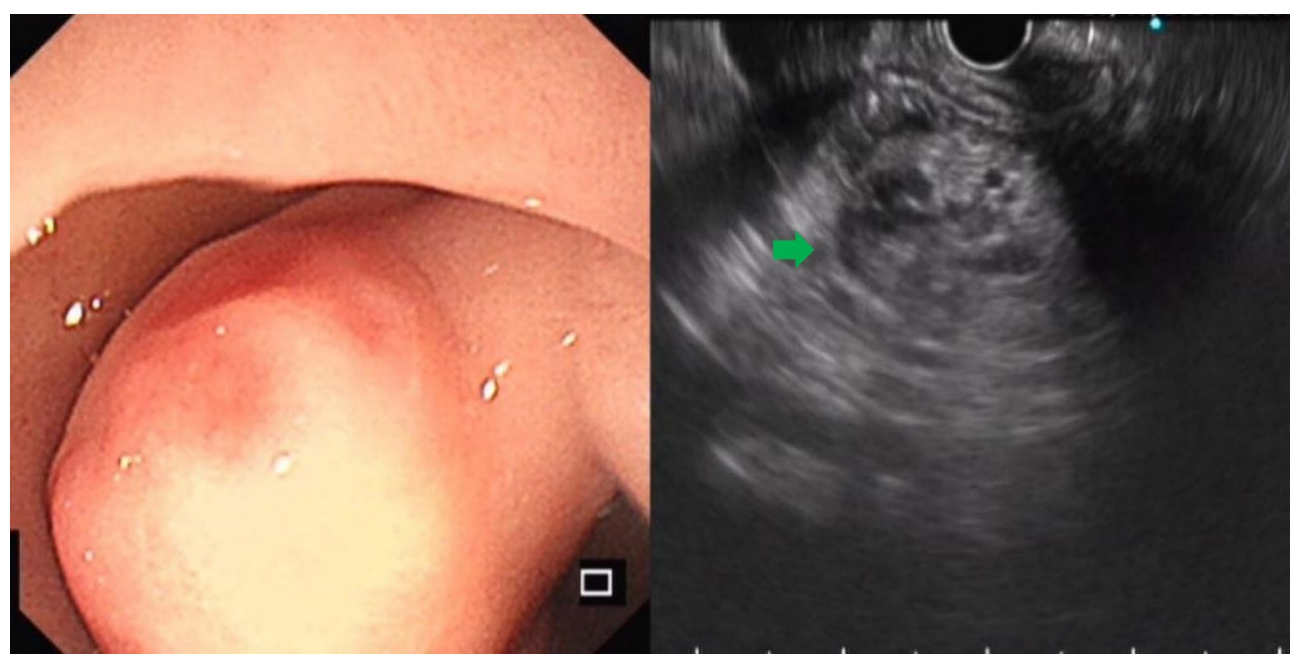

Fig. 7 Hamartoma of duodenal bulb (green arrow)

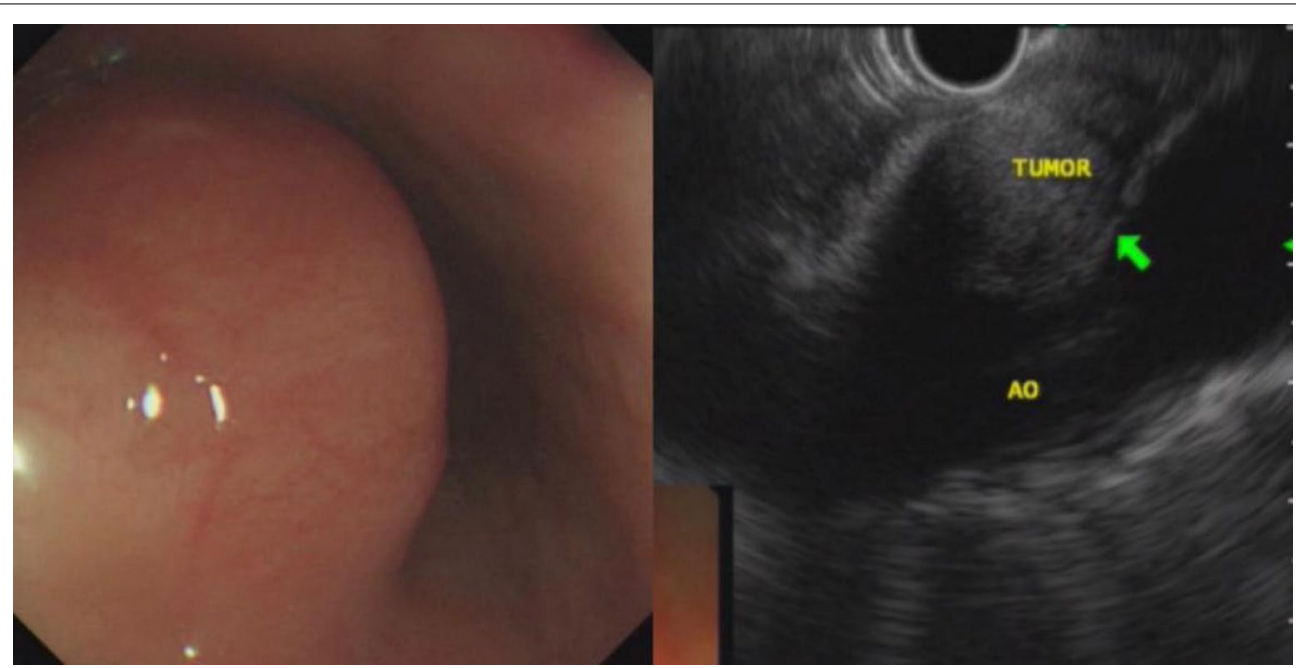

Fig. 8 Solitary fibroma of esophagus (green arrow)

reference for differentiation between the two types of SMLs. Moreover, we calculated the sensitivity, specificity, positive predictive value and negative predictive value in distinguishing these two diseases according to the originating layer of the tumor. The results showed that if the lesion was originating from the muscularis propria, then the lesion was more likely to be a stromal tumor, but if the lesion originated from the muscularis mucosa, it was more likely to be a leiomyoma. Therefore, the differential diagnosis of leiomyoma or stromal tumor could be obtained according to the originating layer of the lesion as identified by EUS.
In this study, stromal tumors mostly appeared as hypoechoic masses originating from the muscularis propria (Fig. 1). It is currently believed that all stromal tumors have malignant potential and differentiation between benign and malignant tumors cannot be made easily [12]. The risk of invasion by stromal tumors has been determined based on parameters such as location, size, mitosis rate and whether the tumor has ruptured [5]. Brand et al. [13] reported that a heterogeneous echotexture greater than $3 \mathrm{~cm}$ and with irregular margins may suggest higherrisk stromal tumors. A study by Jeon and colleagues [14] found that the presence of mucosal ulceration suggests a 


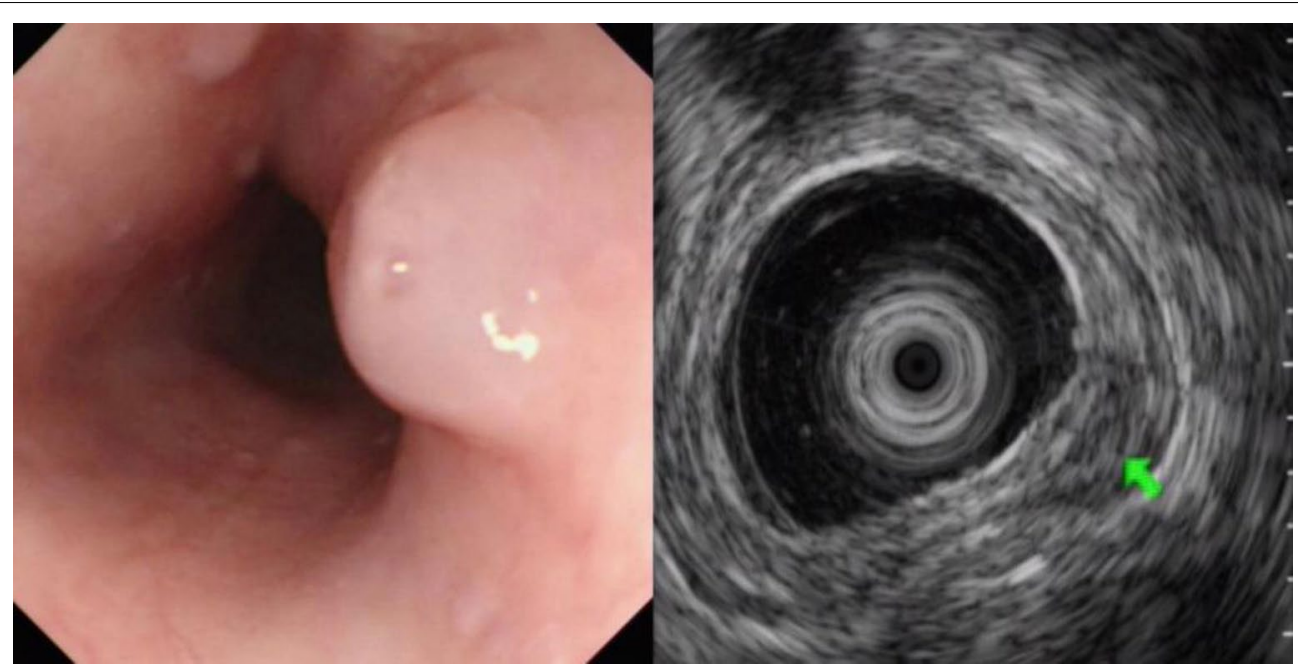

Fig. 9 Haemo-lymphangioma of esophagus (green arrow)

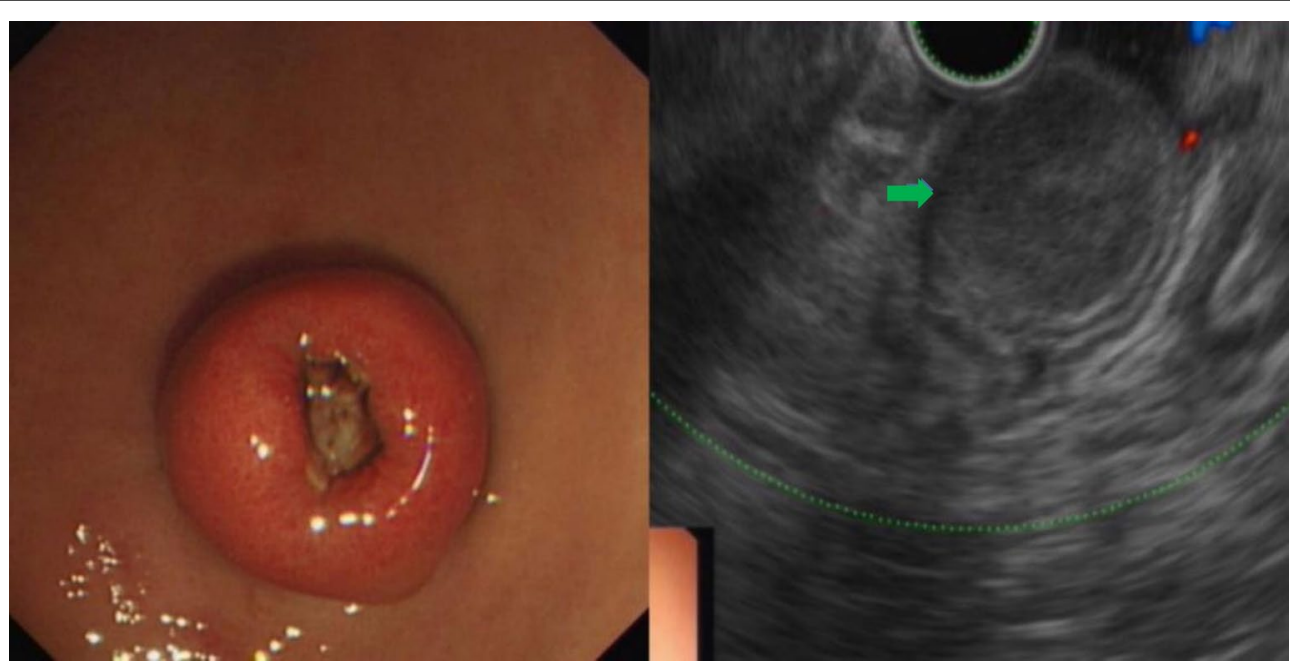

Fig. 10 Angiogenic tumor of gastric antrum (green arrow)

higher risk of malignancy. We compared the EUS characteristics of stromal tumors between the VLR+LR and the IR + HR groups, and found that stromal tumors with a diameter greater than $3 \mathrm{~cm}$ or a surface ulcer on EUS were more likely to be of intermediate or high risk.

Leiomyomas were found to originate primarily in the muscularis mucosa. They appeared as homogeneous or non-homogeneous hypoechoic masses with clear boundaries on EUS, similar to the findings of Codipilly et al. [15] (Fig. 2). A study by Park and colleagues [16] found that $71 \%$ of gastric schwannomas exhibited echogenicity similar to the muscularis propria. In this study, schwannomas mostly manifested as heterogeneous hypoechoic or mixed-echogenic masses originating from the muscularis propria, with clear boundaries and poor blood flow signals (Fig. 3). Chen et al. [17] reported that the EUS features of heterotopic pancreas include indistinct margins, heterogeneous echogenicity (mainly hypoechoic accompanied by small, scattered hyperechoic areas), and duct-like structures with or without echoes. In our study, the heterotopic pancreases were mostly heterogeneous, mixed-to-low-echogenic masses originating from the submucosal layer, and occasionally presented with a luminal-like structure (Fig. 4). Other rare SMLs found in this study included benign cysts (Fig. 5), glomus tumors (Fig. 6), hamartoma (Fig. 7), solitary fibromas 
Table 3 Originating layers of the lesions on endoscopic ultrasonography (EUS)

\begin{tabular}{|c|c|c|c|c|c|}
\hline Pathological results & $\begin{array}{l}\text { Muscularis mucosa } \\
\text { layer }\end{array}$ & Submucosal layer & Muscularis propria layer & Total & $p$ \\
\hline Stromal tumor & 6 & - & 101 & 107 & $0.001^{* *}$ \\
\hline Leiomyoma & 42 & - & 33 & 75 & $0.001^{* *}$ \\
\hline Heterotopic pancreas & 1 & 11 & 1 & 13 & $0.001^{* *}$ \\
\hline Schwannoma & - & 1 & 10 & 11 & 0.094 \\
\hline Inflammation & 3 & - & 7 & 10 & 0.836 \\
\hline Benign cyst & 1 & - & 3 & 4 & 1.000 \\
\hline Glomus tumor & - & - & 4 & 4 & 0.663 \\
\hline Angiolipoma & - & - & 3 & 3 & 1.000 \\
\hline Hamartoma & - & - & 1 & 1 & 1.000 \\
\hline Solitary fibroma & - & - & 1 & 1 & 1.000 \\
\hline Haemo-lymphangioma & 1 & - & - & 1 & 1.000 \\
\hline Angiogenic tumor & - & - & 1 & 1 & 1.000 \\
\hline Total & 54 & 12 & 165 & 231 & \\
\hline
\end{tabular}

**Mmuscularis mucosa layer, the submucosal layer, and the muscularis propria layer have significant differences at the levels of $p<0.01$

Table 4 Diagnostic rates of endoscopic ultrasonography (EUS) diagnosis based on final histopathology of the lesions

\begin{tabular}{lllll}
\hline Pathological result & $\begin{array}{l}\text { Number of pathological } \\
\text { diagnoses }\end{array}$ & $\begin{array}{l}\text { Number of EUS } \\
\text { diagnoses }\end{array}$ & $\begin{array}{l}\text { Number of consistent diagnoses } \\
\text { between pathology and EUS }\end{array}$ & $\begin{array}{l}\text { Diagnostic } \\
\text { consistency } \\
\text { rate }\end{array}$ \\
\hline Stromal tumor & 107 & 147 & 86 & $80.4 \%$ \\
Leiomyoma & 75 & 82 & 51 & $68.0 \%$ \\
Heterotopic pancreas & 13 & 1 & - & $7.7 \%$ \\
Schwannoma & 11 & - & - & 0 \\
Inflammation & 10 & - & - & 0 \\
Benign cyst & 4 & - & - & 0 \\
Glomus tumor & 4 & - & - & 0 \\
Angiolipoma & 3 & - & - & 0 \\
Hamartoma & 1 & - & - & 0 \\
Solitary fibroma & 1 & - & - & 0 \\
Haemo-lymphangioma & 1 & - & 138 & 0 \\
Angiogenic tumor & 1 & - & & $59.7 \%$ \\
Total & 231 & 231 & & 0 \\
\hline
\end{tabular}

(Fig. 8), haemo-lymphangioma (Fig. 9), angiogenic tumor (Fig. 10) and angiolipoma. Multicenter studies with larger sample sizes are required to analyze the EUS characteristics of these uncommon lesions.

This study has some limitations. Firstly, it was a singlecenter, retrospective study. Secondly, the role of EUSFNA/FNB, elastography, or contrast-enhanced EUS in the differentiation between leiomyoma and stromal tumors could not be determined due to the small number of cases. Currently, there are few reports on the role of EUS elastography in the diagnosis of SML $[18,19]$. Several clinical studies have reported the diagnostic value of contrast-enhanced EUS for SML [20, 21]. One study by
Kamata et al. showed that hyper-enhancement and inhomogeneous enhancement were found to be characteristic features of stromal tumors [22]. In the current study, there were too few cases to draw any meaningful conclusions. Future larger prospective studies are needed for additional analysis.

In conclusion, EUS has good diagnostic value for the diagnosis of upper gastrointestinal SMLs. It may be helpful to identify common SMLs based on the size of the lesion and the originating layer as identified by EUS. Stromal tumors with a diameter larger than $3 \mathrm{~cm}$ and a surface ulcer on EUS are more likely to be of intermediate or high risk for malignant potential. EUS 
Table 5 Comparison of diagnosis by endoscopic ultrasonography (EUS) with pathological diagnosis

\begin{tabular}{|c|c|c|c|}
\hline & $\begin{array}{l}\text { Comparison of diagnosis on EUS with } \\
\text { pathological diagnosis }\end{array}$ & & $p$ \\
\hline & Consistent $(n=138)$ & Not consistent $(n=93)$ & \\
\hline Age & $53.67 \pm 11.47$ & $49.29 \pm 11.99$ & 0.407 \\
\hline Mean diameter of lesions measured by EUS & $17.83 \pm 14.68$ & $18.25 \pm 13.49$ & 0.823 \\
\hline $\begin{array}{l}\text { Mean diameter of lesions based on measurement of } \\
\text { surgical specimens }\end{array}$ & $23.25 \pm 19.05$ & $19.69 \pm 14.13$ & 0.152 \\
\hline \multicolumn{4}{|l|}{ Gender } \\
\hline Male & 78 & 44 & \multirow[t]{2}{*}{0.169} \\
\hline Female & 60 & 49 & \\
\hline \multicolumn{4}{|l|}{ Distribution } \\
\hline Esophagus & 47 & 16 & \multirow[t]{3}{*}{0.105} \\
\hline Stomach & 86 & 73 & \\
\hline Duodenum & 5 & 4 & \\
\hline \multicolumn{4}{|l|}{ Origin } \\
\hline Muscularis mucosa layer & 45 & 9 & \multirow[t]{3}{*}{$0.000^{* * *}$} \\
\hline Submucosal layer & 1 & 11 & \\
\hline Muscularis propria layer & 92 & 73 & \\
\hline
\end{tabular}

***Consistent diagnosis group and the inconsistent diagnosis group have significant differences at the level of $p<0.001$

is reliable for the diagnosis of lesions from the muscularis mucosa. However, the diagnosis of heterotopic pancreas, inflammation, benign cyst, glomus tumor,

Table 6 Comparison of the endoscopic ultrasonography (EUS) characteristics of stromal tumors and their categorization into one of two groups based on the risk of invasion

\begin{tabular}{|c|c|c|c|}
\hline EUS characteristic & $\begin{array}{l}\text { VLR + LR } \\
(n=96)\end{array}$ & $I R+H R(n=11)$ & $p$ \\
\hline \multicolumn{4}{|c|}{ Diameter measured under EUS } \\
\hline$\leq 3 \mathrm{~cm}$ & 75 & 1 & $0.000^{* * *}$ \\
\hline$>3 \mathrm{~cm}$ & 21 & 10 & \\
\hline \multicolumn{4}{|l|}{ Surface ulcer } \\
\hline Yes & 17 & 7 & $0.002^{* *}$ \\
\hline No & 79 & 4 & \\
\hline \multicolumn{4}{|l|}{ Clear boundary } \\
\hline Yes & 63 & 8 & 0.747 \\
\hline No & 33 & 3 & \\
\hline \multicolumn{4}{|l|}{ Regular shape } \\
\hline Yes & 46 & 4 & 0.467 \\
\hline No & 50 & 7 & \\
\hline \multicolumn{4}{|l|}{ Echo intensity } \\
\hline Low & 92 & 10 & 0.425 \\
\hline Not low & 4 & 1 & \\
\hline \multicolumn{4}{|l|}{ Echo heterogeneity } \\
\hline Yes & 27 & 1 & 0.282 \\
\hline No & 69 & 10 & \\
\hline
\end{tabular}

$V L R$, very low risk; $L R$, low risk; IR, intermediate risk; $H R$, high risk ** and ***VLR + LR group and the IR + HR group have significant differences at the levels of $p<0.01$ and $p<0.001$, respectively hamartoma, solitary fibroma, haemo-lymphangioma, angiogenic tumor and angiolipoma by EUS is more difficult due to the lack of characteristic features and rarity of these diseases.

\section{Acknowledgements}

This research was funded by the New Xiangya Talent Project of the Third xiangya hosipital of Central South University (20180304), Hunan Provincial Natural Science Foundation of China (2020JJ4853), Hunan Provincial Clinical Medical Technology Innovation Guidance Project (2020SK53616), Scientific Research Project of Hunan Provincial Health Commission (202103032097) and the Independent Exploration and Innovation Project of Central South University (2020zzts295).

\section{Authors' contributions}

Fen Wang had full access to all the data in the study and take responsibility for the integrity of the data and accuracy of the data analysis. Qian Su and Jin Peng wrote the main manuscript text and Qian Su prepared Figs. 1, 2, 3, 4, 5 and 6. All authors read and approved the final manuscript.

\section{Funding}

No funding has been received.

Availability of data and materials

All other data used to generate tables for the study are available upon request by the corresponding author.

\section{Declarations}

\section{Ethics approval and consent to participate}

Approval for this study was obtained from the Ethics Committee of The Third Xiangya Hospital of Central South University (reference number: 2021-S198). All experiments were performed in accordance with relevant guidelines and regulations. Written informed consent was obtained from individual or guardian participants.

\section{Consent for publication}

Not applicable. 


\section{Competing interests}

The authors declare that they have no competing interests.

\section{Author details}

1 Department of Gastroenterology, Hunan Key Laboratory of Non-Resolving Inflammation and Cancer, The Third Xiangya Hospital of Central South University, 138 Tongzi Road, Changsha 410013, Hunan, People's Republic of China. ${ }^{2}$ First Hospital of Changsha, 311 Yinpan Road, Changsha 410005, Hunan, People's Republic of China.

Received: 18 June 2021 Accepted: 27 September 2021

Published online: 07 October 2021

\section{References}

1. Gress FG. Progress in endoscopic ultrasonography. Gastrointest Endosc Clin N Am. 2017:27(4):15-6.

2. Gong EJ, Kim DH. Endoscopic ultrasonography in the diagnosis of gastric subepithelial lesions. Clin Endosc. 2016:49(5):425-33.

3. Dias D, Magalhães J, Monteiro S, et al. The role of endoscopic ultrasound in the diagnostic assessment of subepithelial lesions of the upper gastrointestinal tract. GE Port J Gastroenterol. 2016;23(6):287-92.

4. Kuroki K, Oka S, Tanaka S, et al. Clinical significance of endoscopic ultrasonography in diagnosing invasion depth of early gastric cancer prior to endoscopic submucosal dissection. Gastric Cancer. 2021;24(1):145-55.

5. Chinese Society Of Clinical Oncology Csco Expert Committee On Gastrointestinal Stromal Tumor. Chinese consensus guidelines for diagnosis and management of gastrointestinal stromal tumor. Chin J Cancer Res. 2017;29(4):281-93.

6. Alvarez-Sanchez MV, Gincul R, Lefort C, et al. Role of contrast-enhanced harmonic endoscopic ultrasound in submucosal tumors. Endosc Ultrasound. 2016:5(6):363-7.

7. Jenssen C, Hocke M, Fusaroli P, et al. EFSUMB guidelines on interventional ultrasound (INVUS), part IV - EUS-guided interventions: general aspects and EUS-guided sampling (short version). Ultraschall Med. 2016;37(2):157-69.

8. Chen T, Hsu C, Chu Y, et al. Association of endoscopic ultrasonographic parameters and gastrointestinal stromal tumors (GISTs): can endoscopic ultrasonography be used to screen gastric GISTs for potential malignancy? Scand J Gastroenterol. 2016;51(3):374-7.

9. Ignee A, Jenssen C, Hocke M, et al. Contrast-enhanced(endoscopic) ultrasound and endoscopic ultrasound elastography in gastrointestinal stromal tumors. Endosc Ultrasound. 2017:6(1):55-60.
10. Attila T, Aydin Ö. Lesion size determines diagnostic yield of EUS-FNA with onsite cytopathologic evaluation for upper gastrointestinal subepithelial lesions. Turk J Gastroenterol. 2018;29(4):436-41.

11. Schulz RT, Fabio LC, Franco MC, et al. Predictive features for histology of gastric subepithelial lesions. Arq Gastroenterol. 2017;54(1):11-5.

12. Boye K, Berner JM, Hompland I, et al. Genotype and risk of tumour rupture in gastrointestinal stromal tumour. Br J Surg. 2018;105(2):e169-75.

13. Brand B, Oesterhelweg L, Binmoeller KF, et al. Impact of endoscopic ultrasound for evaluation of submucosal lesions in gastrointestinal tract. Dig Liver Dis. 2002;34(4):290-7.

14. Jeon SW, Park YD, Chung YJ, et al. Gastrointestinal stromal tumors of the stomach: endosonographic differentiation in relation to histological risk. J Gastroenterol Hepatol. 2007;22(12):2069-75.

15. Codipilly DC, Fang $\mathrm{H}$, Alexander JA, et al. Subepithelial esophageal tumors: a single-center review of resected and surveilled lesions. Gastrointest Endosc. 2018:87(2):370-7.

16. Park HC, Son DJ, Oh HH, et al. Endoscopic ultrasonographic characteristics of gastric schwannoma distinguished from gastrointestinal stromal tumor. Korean J Gastroenterol. 2015;65(1):21-6.

17. Chen $\mathrm{SH}$, Huang $\mathrm{WH}$, Feng $\mathrm{CL}$, et al. Clinical analysis of ectopic pancreas with endoscopic ultrasonography: an experience in a medical center. J Gastrointest Surg. 2008;12(5):877-81.

18. Tsuji Y, Kusano C, Gotoda T, et al. Diagnostic potential of endoscopic ultrasonography-elastography for gastric submucosal tumors: a pilot study. Dig Endosc. 2016;28(1):173-8.

19. Azuma M, Kusano C, Gotoda T. Diagnostic potential of endoscopic ultrasonography-elastography for gastric submucosal tumors. Dig Endosc. 2015;5(2):23.

20. Park HY, Jeon SW, Lee HS, et al. Can contrast-enhanced harmonic endosonography predict malignancy risk in gastrointestinal subepithelial tumors? Endosc Ultrasound. 2016:5(6):384-9.

21. Zhao Y, Qian L, Li P, et al. The diagnostic value of endoscopic ultrasonography and contrast-enhanced harmonic endoscopic ultrasonography in gastrointestinal stromal tumors. Endosc Ultrasound. 2016;5(2):111-7.

22. Kamata K, Takenaka M, Kitano M, et al. Contrast-enhanced harmonic endoscopic ultrasonography for differential diagnosis of submucosal tumors of the upper gastrointestinal tract. J Gastroenterol Hepatol. 2017:32(10):1686-92

\section{Publisher's Note}

Springer Nature remains neutral with regard to jurisdictional claims in published maps and institutional affiliations.
Ready to submit your research? Choose BMC and benefit from:

- fast, convenient online submission

- thorough peer review by experienced researchers in your field

- rapid publication on acceptance

- support for research data, including large and complex data types

- gold Open Access which fosters wider collaboration and increased citations

- maximum visibility for your research: over 100M website views per year

At $\mathrm{BMC}$, research is always in progress.

Learn more biomedcentral.com/submissions 